\title{
Tracking Misplaced Objects Using Bluetooth and GPS with Arm Cortex M3 Development Board \\ ${ }^{* 1}$ D. Aswani, ${ }^{2}$ P. Chandramouli, \\ ${ }^{1,2}$ ECE Department, SVEW, Tirupathi.

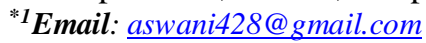

Received: 06th October 2017 Accepted: 14th November 2017, Published: 31st December 2017

\begin{abstract}
In this busy and computerized world it may not be possible to remind all those small stuff where we kept. We have to make things small and comfortable. The object is may be a car key, pen drive or mobile, it often important to carry with us, what will happen if we forgot it somewhere. It may be a small thing but it may very important. When you're having a difficult time finding a specific folder or file on your computer, you simply run a quick search and you've found what you're looking for. What if you could run a similar search, but this time you want to determine if you remembered maybe track down those long lost keys of yours? Our paper making that type of detection possible with "Tracking Misplaced Objects Using Bluetooth and GPS with ARM Cortex M3 Development Board".
\end{abstract}

Keywords: ARM CortexM3,GPS, Wireless Tag, UART

\section{Introduction}

In this project the device using the Tracking Misplaced Things is to be used in a daily life to get easy finding their misplaced things. The system also has to be able to store the location of the object with the help of GPS adapter. It is to find misplaced things such as car keys. A wireless tag(either using bluetooth or $\mathrm{WiFi}$ ) attached to your important things which in this case, a car key can send a signal to your ARM Cortex based system with wi-fi or Bluetooth support. Whenever the tag
Misplaces, the ARM controller will take suitable action. The microcontroller will continuously monitor the signal from the "tagged" object. Whenever the strength of the received signal goes below the threshold strength this will indicate that the tagged object has been misplaced by its owner. Upon noticing the signal going below threshold value the microcontroller will record its last known position with the help of a GPS adapter at a point where it has received good signal strength from the tag.

\section{Proposed System}

This chapter describes the various requirements for the processing hardware. The device using the Tracking Misplaced Things is to be used in a daily life to get easy finding their misplaced things. The system also has to be able to store the location of the object with the help of GPS adapter.

Fig 1: Proposed system

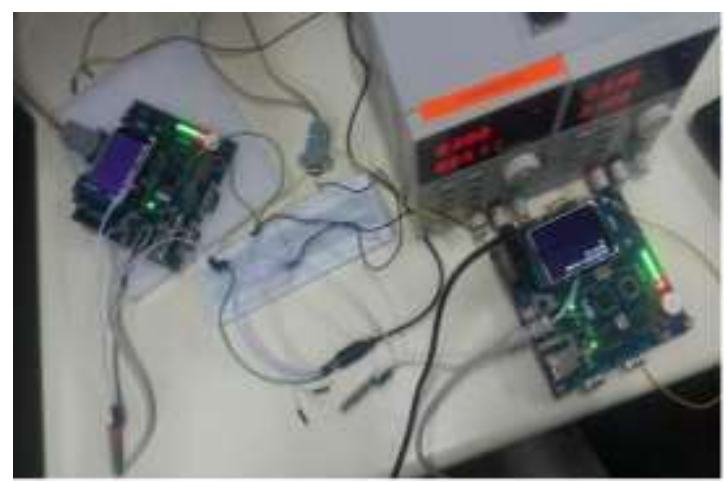


Interfacing of UART on board with Desktop Both Bluetooth and GPS are interfaced with the LPC1768 using UART.

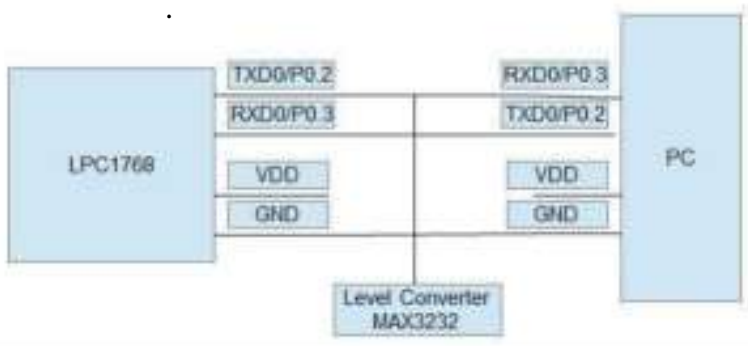

Fig 2: Connections between LPC1768 and PC through UART

This is the final output of UART

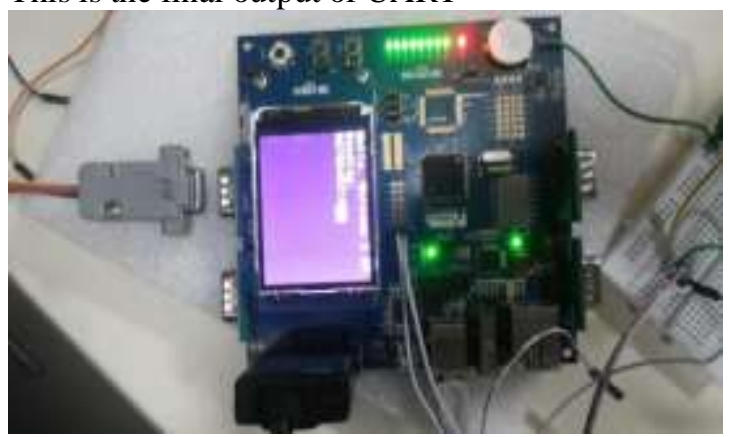

Fig 3: UART Output

Interfacing of Bluetooth with LPC1768

To give AT Commands to the Bluetooth it must be connected to pc's UART PORT. But since Bluetooth works at CMOS Level a level converter is needed.

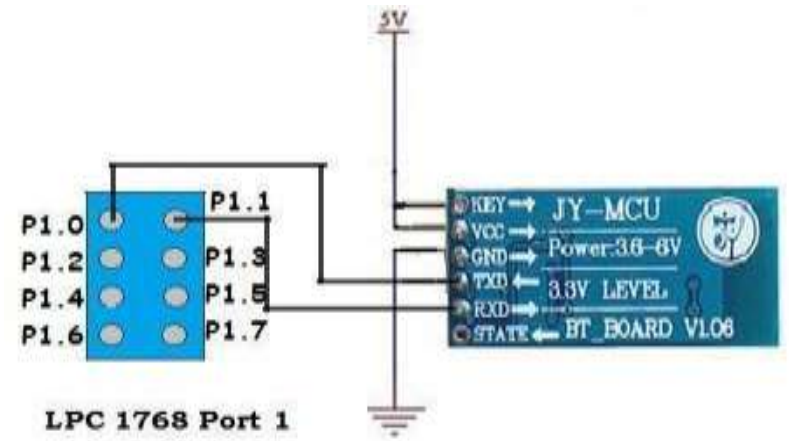

Fig 4: Interfacing Bluetooth with LPC1768

We have to apply $3 \mathrm{~V}$ to key for giving AT Commands. $3 \mathrm{~V}$ supply is needed for powering it on.
TXD is connected to RXD1 of LPC1768. RXD is connected to TXD1 of LPC1768. First of all we have to set the Baud rate to 38400 in the windows terminal. No parity, 1 stop bit and 8 data bits.

To search nearby Bluetooth devices for their address. Its response should be as follows:

+INQ:2013:10:154336 -> Slave BT Address

We can actually link with Slave Type:

AT+LINK=2013,10,154336.

Its response must be $\mathrm{OK}$.

Connecting GPS to PC through UART

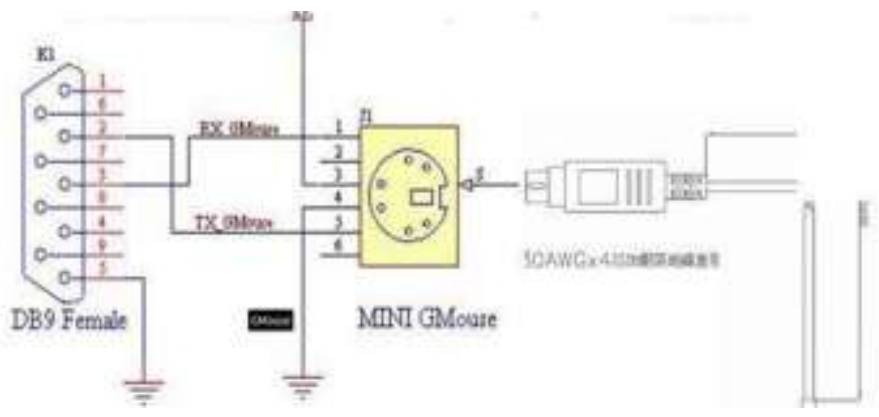

Fig 5: Interfacing Mini GPS to PC through UART

In order to receive data from GPS into our Microcontroller LPC1768, following connections should be made:-

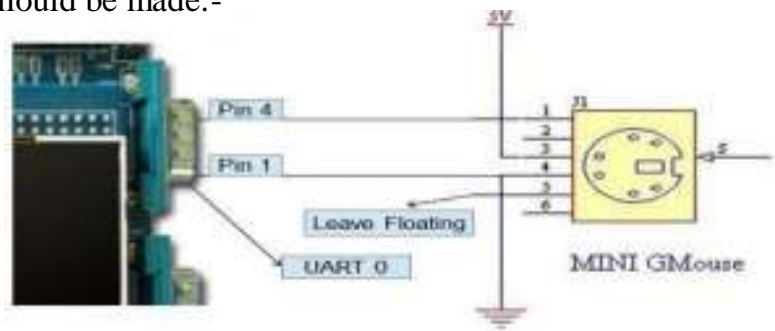

Fig6: Interfacing GPS with LPC1768

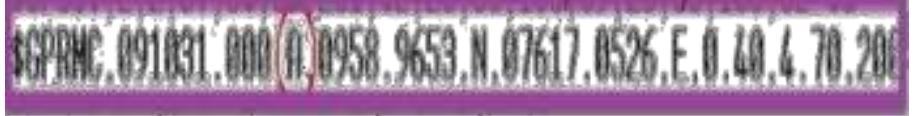

Fig7: GPS output format

The final GPS output format contains recommended navigation information. Here ' $A$ ' represents the 'valid data' and ' $V$ ' represents the 'Not valid data'. It has the latitude and longitude values. Here N,S,E,W are North, South, East, West respectively. 


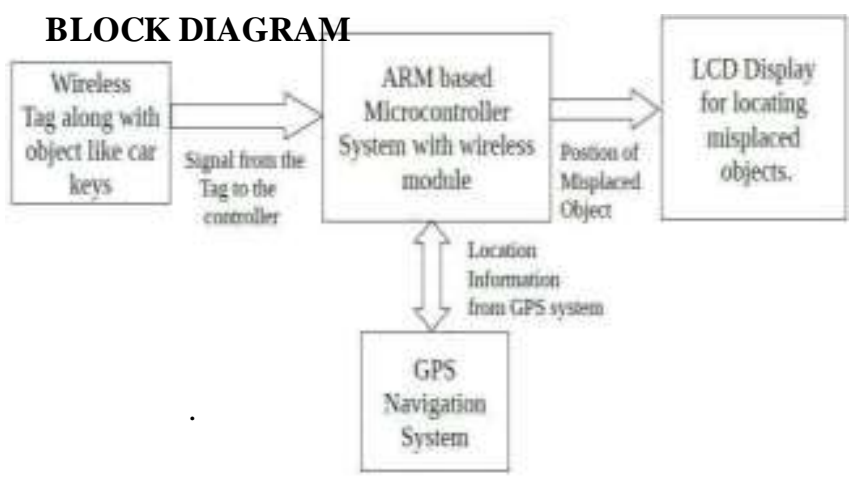

Fig 8: Block Diagram

It has wireless tag, ARM Controller, LCD Display and GPS Navigation system. Wireless tag is along with the object like car keys and important things. ARM Controller system is along with wireless module. It controls the functionality among Wireless tag, LCD Display, GPS Navigation system.

We can find the location of misplaced things by using the GPS Navigation system. LCD Display is used for locating the misplaced objects. The wireless tag either uses Bluetooth or Wi-Fi. Whenever the tag misplaces, the ARM Controller will give the location, where the thing placed with the help of GPS. GPS and wireless tag will work with 5v supply.

\section{HARDWARE MODULES ARM CORTEX M-3:}

The LPC1768 is an ARM Cortex-M3 based microcontroller for embedded applications. It requires a high level of integration and low power dissipation. The Cortex-M3 processor is a high performance 32-bit processor designed for the microcontroller market. It offers significant benefits to developers, including:

- $\quad$ Outstanding processing performance combined with fast interrupt handling
- Enhanced system debug with extensive breakpoint and trace capabilities

- Efficient processor core, system and memories

- Ultra-low power consumption with integrated sleep mode and an optional deep sleep mode

- Platform security robustness, with an optional integrated Memory Protection Unit (MPU).

\section{WIRELESS TAG (BLUETOOTH):}

Wireless networking is a hot industry segment, several wireless technologies have been targeted primarily for data transmission. Bluetooth is a standard used to build small networks between peripherals: a form of "wireless wires".

Most of the people in the industry are familiar with the hype surrounding Bluetooth, though it seems to have died down as real devices have been brought to market. Bluetooth is a way for devices to wirelessly communicate over short distances. Wireless communication has been around since the late nineteenth century, and has taken form in radio, infrared, television, and more recently 802.11.

What distinguishes Bluetooth is its special attention to short-distance communication, usually less than 30 $\mathrm{ft}$. Both hardware and software are affected by this special attention. Here we are using JY-MCU Bluetooth module. This is a Bluetooth Wireless module that provides a simple interface for connecting to LPC1768.

The module provides a method to connect wirelessly with a PC or Bluetooth phone to transmit/receive embedded data such as GPS data, ADC voltage reading and other parameters. HC-05 embedded Bluetooth serial communication module (can be short 
for module) has two work modes: order-response work mode and automatic connection work mode. And there are three work roles (Master, Slave and Loopback) at the automatic connection work mode. When the module is at the automatic connection work mode, it will follow the default way set lastly to transmit the data automatically.

\section{GPS NAVIGATION SYSTEM:}

GPS based tracking system will provide effective, real time object location, and reporting. A GPS based tracking system will inform where your vehicle is and where it has been, how long it has been. The system uses geographic position and time information from the Global Positioning Satellites. The system has an "On- Board Module" which resides in the object to be tracked.

The On-Board module consists of GPS receiver and ARM processor. During object motion, its real-time parameters such as location are stored in EEPROM. The system takes advantage of wireless technology in providing powerful management transportation engine. The use of GSM and GPS technologies allows the system to track object and provides the most up-to-date information about ongoing trips. This system finds its application in real time traffic surveillance. It could be used as a valuable tool for real time traveler information, congestion monitoring, and system evaluation.

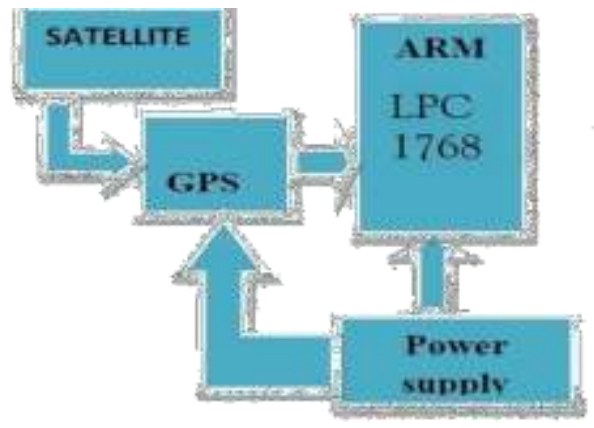

Fig9: Block diagram of tracking system
Microcontroller unit form the heart of tracking unit, which acquires and process the position data from the GPS module. The GPS receiver of object terminal receives and resolves the navigation message broadcasted by GPS position satellites, computes the longitude and latitude of object coordinates.

\section{Software Design Tools KEIL SOFTWARE}

Keil compiler is software used where the machine language code is written and compiled. After compilation, the machine source code is converted into hex code which is to be dumped into the microcontroller for further processing. Keil compiler also supports $\mathrm{C}$ language code.

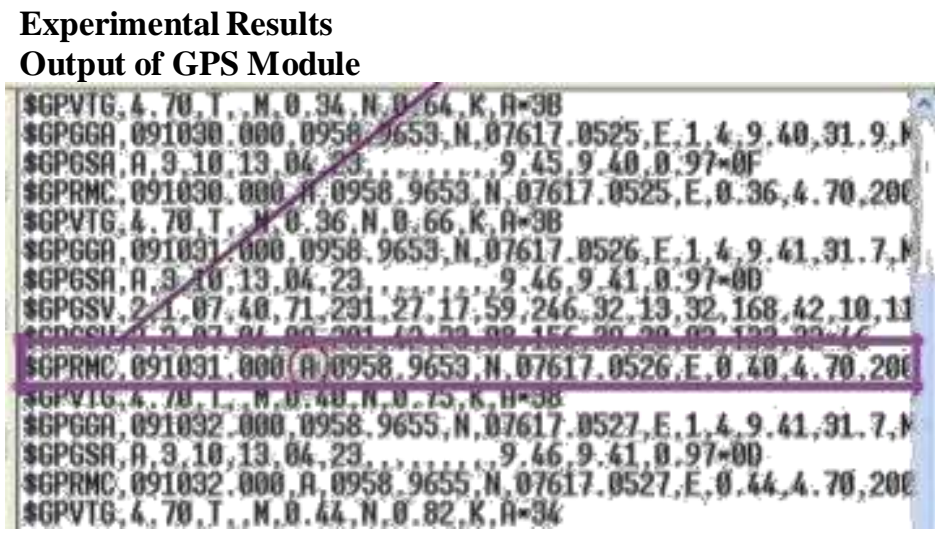

Fig 10: GPS Output at hyper terminal

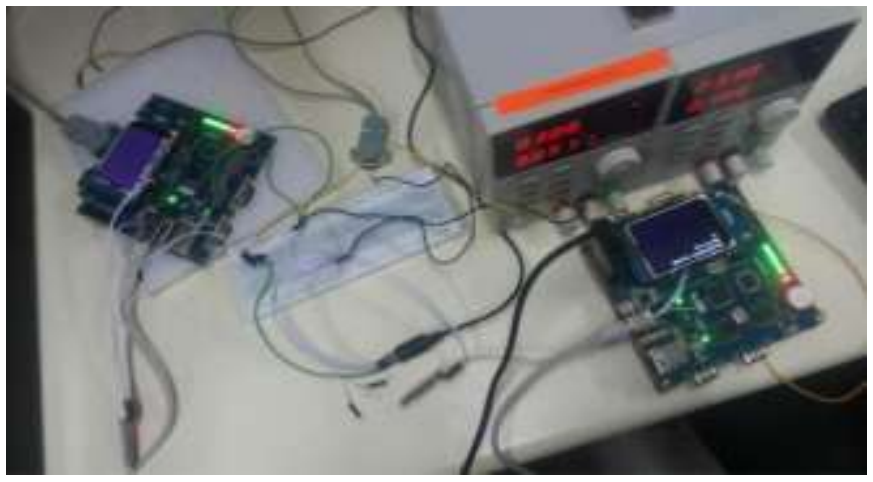

Fig 11: BT modules communicating each other 


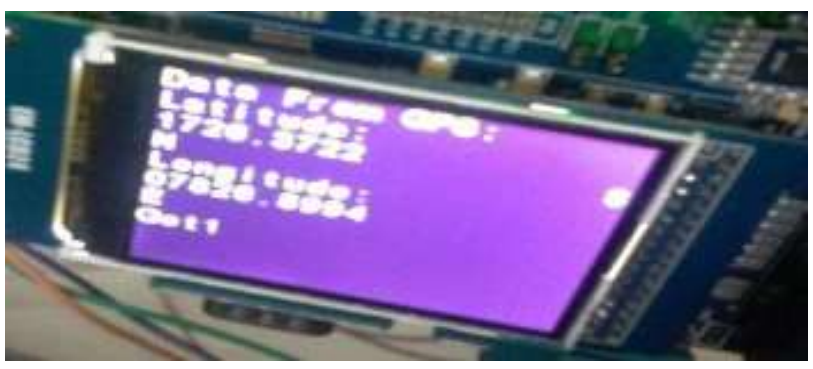

Fig12: UART and GPS Output

\section{Conclusion}

This version of our project will track position of the objects in terms of latitude and longitude when it is misplaced.

People who always face the problem of finding their keys and other belongings benefit from an electronic device that helps them find their missing items

In this project we used Bluetooth as my wireless tag because of its less cost, less power consumption, but in this rapid world everything growing fast, rapid changes coming in to existence, so definitely we can implement it with advanced technologies like Wi-fi and ZigBee. Integrate this project into an android app which can further enhance the usefulness.

\section{References}

[1] K. Rangan, T.Vigneswaran, An Embedded Systems Approach to Monitor Green House, IEEE conference 2010, 978-1-4244-9182-7/10

[2] Wei Dongshan, Complete Guide to Embedded Linux Application Development, Post

[3] Positioning And Navigation System Using GPS,IEEE Conference by J.Parthasarathy, Member Technical Staff, Sun Microsystems Pvt Itd, India, Divyasree chambers, off-Langford road, Bangalore-560027, India.

[4] International Journal of Computer Applications (0975 - 8887) Volume 109 - No. 8, January 2015, Global Positioning System for Object Tracking, Abha Damani Integrate M.Sc. (IT), Uka Tarsadia University, Bardoli, Gujarat.

\section{Acknowledgment}

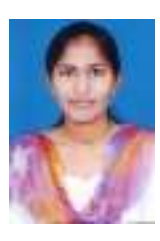

Author1: D. Aswani is working as Assistant Professor in the department of ECE, SVEW, Tirupati. She published one International .She has done her PG Diploma in Electronic product design at CDAC, Hyd.

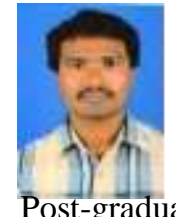

Author 2: Mr. P.Chandra Mouli received his graduation degree in ECE from Anamacharya Institutions of Technology and Science Rajampet, Post-graduation degree in Embedded Systems from SVCET, Chittoor and $\mathrm{He}$ presently working as Assistant Professor at S. V. Engineering College for Women, Tirupathi. 